\title{
Slip and no-slip temperature boundary conditions at interface of porous, plain media: conduction
}

\author{
M. SAHRAOUI and M. KAVIANY \\ Department of Mechanical Engineering and Applied Mechanics, \\ The University of Michigan, Ann Arbor, MI 48109, U.S.A.
}

(Received 24 June 1991 and in final form 24 February 1992)

\begin{abstract}
The phase distribution nonuniformities near bounding surfaces result in anisotropy and nonuniformity of the effective thermal conductivity tensor. For a two-dimensional porous medium made of cylindrical particles, we evaluate the properties of this tensor for cases where the medium is bounded by the fuid saturating it or by a solid surface. The use of a uniform effective conductivity, such as the bulk (far from the surface) value, along with the near surface temperature distribution results in an error in the calculated heat flux. We examine this error and the errors resulting from the use of other approximations of the effective conductivity near the surface. We also point out a slip in the surface temperature occurring when the bulk effective conductivity and the temperature distribution away from the surface are used to extrapolate the temperature at the interface. A slip coefficient is used to account for this slip in temperature.
\end{abstract}

\section{INTRODUCTION}

UNDER the assumption of local thermal equilibrium, a single energy equation can be written for solidfluid heterogeneous systems such as saturated porous media. This single energy equation includes a local effective thermal conductivity tensor $\mathbf{K}_{\mathbf{c}}$ which represents a local volume-averaged molecular conduction through both phases and a local dispersion tensor $\mathbf{D}^{\text {d }}$ which represents the local volume-averaged hydrodynamic dispersion. This dispersion results from the simultaneous presence of a temperature and a velocity gradient within the pore. Because of the anisotropy and nonuniformity of the solid matrix structure, both $\mathbf{K}_{\mathrm{c}}$ and $\mathbf{D}^{\mathrm{d}}$ are in general anisotropic and nonuniform. In this paper we examine the variation of $\mathbf{K}_{\mathrm{c}}$ near the bounding surface and for a two-dimensional structure. In a later paper we will combine the hydrodynamic analysis, already reported by Sahraoui and Kaviany [1], with a heat transfer analysis in order to obtain the variation of $\mathbf{D}^{\mathbf{d}}$.

Figure $1(a)$ is a rendering of the phase distributions near a solid bounding surface where the conductivity of this solid $k_{s_{\mathrm{b}}}$ can be different to the solid particle and the fluid conductivities $k_{\mathrm{s}}$ and $k_{\mathrm{r}}$. The heat flows from the bound medium (here a solid) to the fluid phase through $A_{\mathrm{s}_{\mathrm{b}} \mathrm{r}}$ and to the solid phase through $A_{\mathrm{s}_{\mathrm{b}} \mathrm{s}}$. We expect each of the three conductivities $k_{\mathrm{s}_{\mathrm{h}}}$, $k_{\mathrm{s}}$, and $k_{\ulcorner}$to influence the magnitude of $\mathbf{K}_{\mathrm{e}}$ at the interface. Also, the distribution of the local porosity significantly influences the magnitude of $\mathbf{K}_{\mathrm{c}}$ causing a nonuniformity. This nonuniformity near the bounding surfaces has been recognized by many investigators such as Yagi and Kunii [2], Ofuchi and Kunii [3] and Matsuura et al. [4]. Ofuchi and Kunii attempted to model the nonuniformity of $\mathbf{K}_{c}$ by including a modification which allows for a larger porosity near the bounding solid surface of a packed bed of spherical particles. This treatment of the interface is called the layered model. In this model the average porosity for the distance of one half of a particle diameter from the boundary is used to evaluate the effective thermal conductivity at the bounding surface. This corresponds to an averaging volume which, although smaller than the representative elementary volume, is too large to represent the pointwise effective conductivity needed for the evaluation of the surface heat flux using the pointwise temperature gradient. The bulk (away from the interface) effective thermal conductivity is also used near the bounding surface, along with the extrapolation of the temperature field away from the boundary. This, in general (and when $k_{\mathrm{r}}<k_{\mathrm{s}}$ ), has resulted in a larger surface heat flux or a surface temperature slip. Then, attempts have been made to model this temperature slip by using a film heat transfer coefficient [4]

The existing rigorous analytical-numerical treatments of the bulk effective conductivity, which are mostly for packed beds of spherical particles, are in general not capable of handling anisotropy and nonuniformity of the particle arrangements near the boundary. The nonuniformity could be modeled using a variable $k_{c \perp}$ and imposing the continuity of the temperature, i.e. no temperature slip is allowed at the boundary. This is given by the steady state, onedimensional equation with negligible radiation effects

$$
\frac{\mathrm{d}}{\mathrm{d} y}\left[k_{\mathrm{c} \perp}(y) \frac{\mathrm{d} T}{\mathrm{~d} y}\right]=0
$$

where $k_{\mathrm{e} \perp}(y)$ is the variable local transverse effective 


\section{NOMENCLATURE}

a side length of square [m]

$A_{\mathrm{f}}$ solid-fluid interfacial area [m $\left.{ }^{2}\right]$

$\mathbf{b}_{r} . \mathbf{b}_{i} \quad$ transformation vector [m]

c thickness of connecting arm between square cylinders [m]

d diameler of circular cylinders [m]

$D^{d} \quad$ dispersion tensor $\left[\mathrm{m} \mathrm{s}^{-2}\right]$

$f$ Cunction used in the correlation of $\mathbf{K}_{\mathbf{c}}$

$q$ bulk effective conductivity correlation

$h \quad$ width of plain medium [m]

I identily lensor

$k$ conductivity [ $\mathrm{W} \mathrm{m}^{-1} \mathrm{~K}^{-1}$ ]

$\mathbf{K}_{r} \quad$ effective conductivity tensor [W $\mathrm{m}^{-1} \mathrm{~K}^{-1}$ ]

$k$ isotropic effective conduclivity

[W m $\left.\mathrm{W}^{-1} \mathrm{~K}^{-1}\right]$

$k_{s_{n}} \quad$ conductivity of solid bounding medium [W $\mathrm{m}^{-1} \mathrm{~K}^{-1}$ ]

$k_{\mathrm{c} \perp}, k_{\mathfrak{c} \mid}$ transverse and longitudinal components of effective conductivity tensor [ $\mathrm{W} \mathrm{m}^{-1} \mathrm{~K}^{-1}$ ]

, cell dimension $[\mathrm{m}]$

$r$ periodicity vector $[\mathrm{m}]$

$\mathbf{n}_{\mathrm{r}}$ unit normal vector outward from fuid phase

$\mathbf{n}_{\mathrm{s}} \quad$ unit normal vector outward from solid phase

q heat flux vector [ $\mathrm{W} \mathrm{m}^{-2}$ ]

$q_{x}, q_{1} \quad x$ - and $y$-components of the heat flux vector [ $\mathrm{W} \mathrm{m}^{-2}$ ]

$q$ xicr heat flux obtained from the exact solution [ $\mathrm{W} \mathrm{m}^{-2}$ ]

$q_{\text {no slip }}$ heal flux based on no temperature slip [W $\mathrm{m}^{-2}$ ]

$r \quad$ radial coordinate [m]

$R \quad$ radius of circular cylinder [m]

$T \quad$ local temperature [K]

$\Delta T \quad$ imposed temperature difference $[\mathrm{K}]$

$T_{\mathrm{i}}^{\prime} \quad$ fluid-phase temperature deviation, $T-\langle T\rangle_{V}^{r} \cdot[\mathrm{K}]$

$T_{h} \quad$ temperature boundary condition at $y=h$

$T_{s}^{\prime} \quad$ solid-phase temperalure deviation. $T-\langle T\rangle_{i} \cdot[\mathrm{K}]$

$T_{\mathrm{s}} \quad$ temperature of solid bounding surface [K]

$\langle T\rangle$, local area-averaged temperature in the $x$-direction,

$\langle T\rangle_{A_{2}}(x, y)=\int_{-0.5}^{0.5} T\left(x+x^{\prime}, y\right) \mathrm{d} x^{\prime}[\mathrm{K}]$ $\langle T\rangle_{\text {A }}$ local area-averaged lemperature in the $y$-direction. $\langle T\rangle_{A_{1}}\left(x, y^{\prime}\right)=\int_{0.5}^{0.5} T\left(x, y^{\prime}+y^{\prime}\right) d_{y^{\prime}}[\mathrm{K}]$

$\langle T\rangle_{1}$ Iocal volume-averaged temperature, ( $/ V) \int_{1} \cdot T \mathrm{~d} V[\mathrm{~K}]$

$\langle T\rangle_{\text {r. }}$ local fluid-phase volume-averaged temperalure. $\left(\mathrm{l} / V_{\mathrm{r}}\right) \int_{\mathrm{r}_{\mathrm{r}}} T_{\mathrm{r}} \mathrm{d} V^{\prime}[\mathrm{K}]$

$\langle T\rangle_{i}$. local solid-phase volume-averaged temperature, $\left(\mathrm{I} / V_{\mathrm{q}}\right) \int_{1}, T_{\mathrm{s}} \mathrm{d} V[\mathrm{~K}]$

$V \quad$ local representative elementary volume $\left[\mathrm{m}^{3}\right]$

$x, y$ Cartesian coordinates [m].

\section{Greek symbols}

$\alpha_{T} \quad$ slip coefficient

E. porosity

() tangential coordinate

$i \quad$ pore-level length scale

$\phi \quad$ volume fraction of the solid, $I-\varepsilon$.

\section{Subscripts}

A area

exact from exact solution

f fluid

$h \quad$ at $y^{\prime}=h$

, at $x=1$

$s$ solid

$s_{h} \quad$ solid bounding wall

$V \quad$ volume

$x \quad x$-direction

$y \quad y$-direction

$\theta$ tangential direction

\| longitudinal

$\perp \quad$ transverse.

\section{Superscripts}

f fluid

s solid

- deviation

$+\quad$ plain medium side

- $\quad$ porous medium side.

Other symbols
\langle\rangle$^{r}$
volume averaged over the fluid phase
\langle\rangle$^{\mathrm{s}}$ volume averaged over the solid phase
\langle\rangle$_{A}$ area averaged in the $x$-direction
\langle\rangle$_{A}$, area averaged in the $y$-direction
\langle\rangle$_{1}$ volume averaged.

conductivity of the medium. This equation applies to both the porous and plain media, but for the former the temperature is written as either the area- or the volume-average (i.e. $\langle T\rangle_{A}$ or $\langle T\rangle_{Y}$ ). In the porous medium and away from the interface, $k_{\mathrm{c} \perp}(y \rightarrow-\infty)$ is a constant and corresponds to the bulk effective conductivity $k_{\mathrm{c}}$ and in the plain medium $k_{\mathrm{c} \perp}(y>0)=k_{\mathrm{f}}$ or $k_{\mathrm{c} \perp}(y>0)=k_{\mathrm{s}_{\mathrm{b}}}$ depending on 
(b)
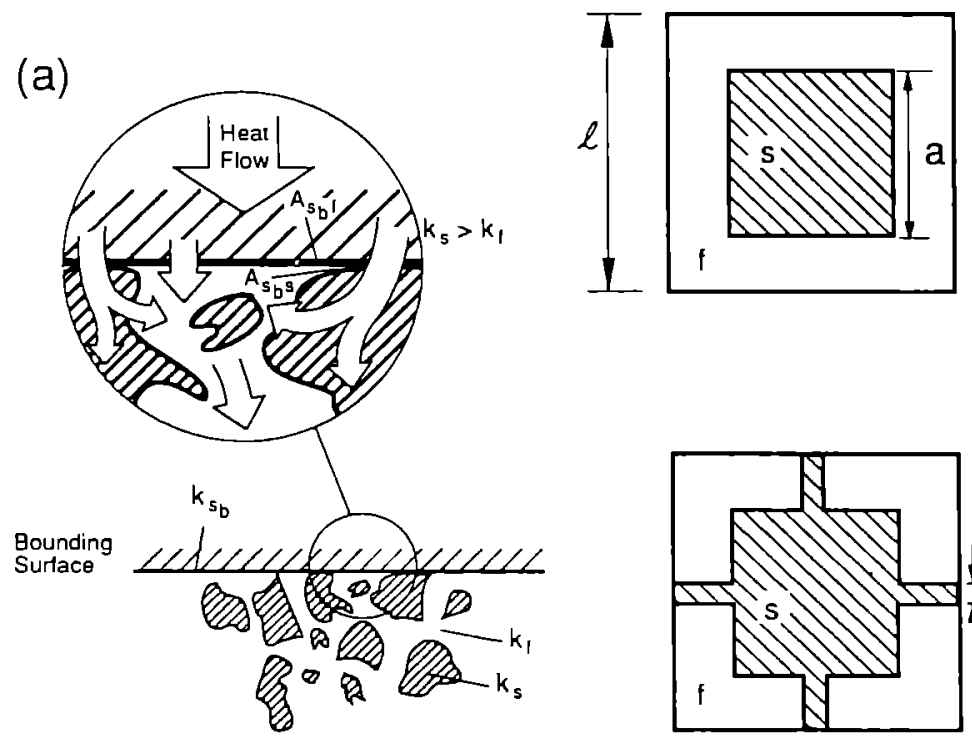

(c)

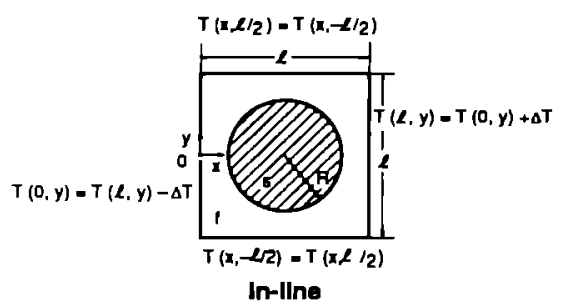

(d)
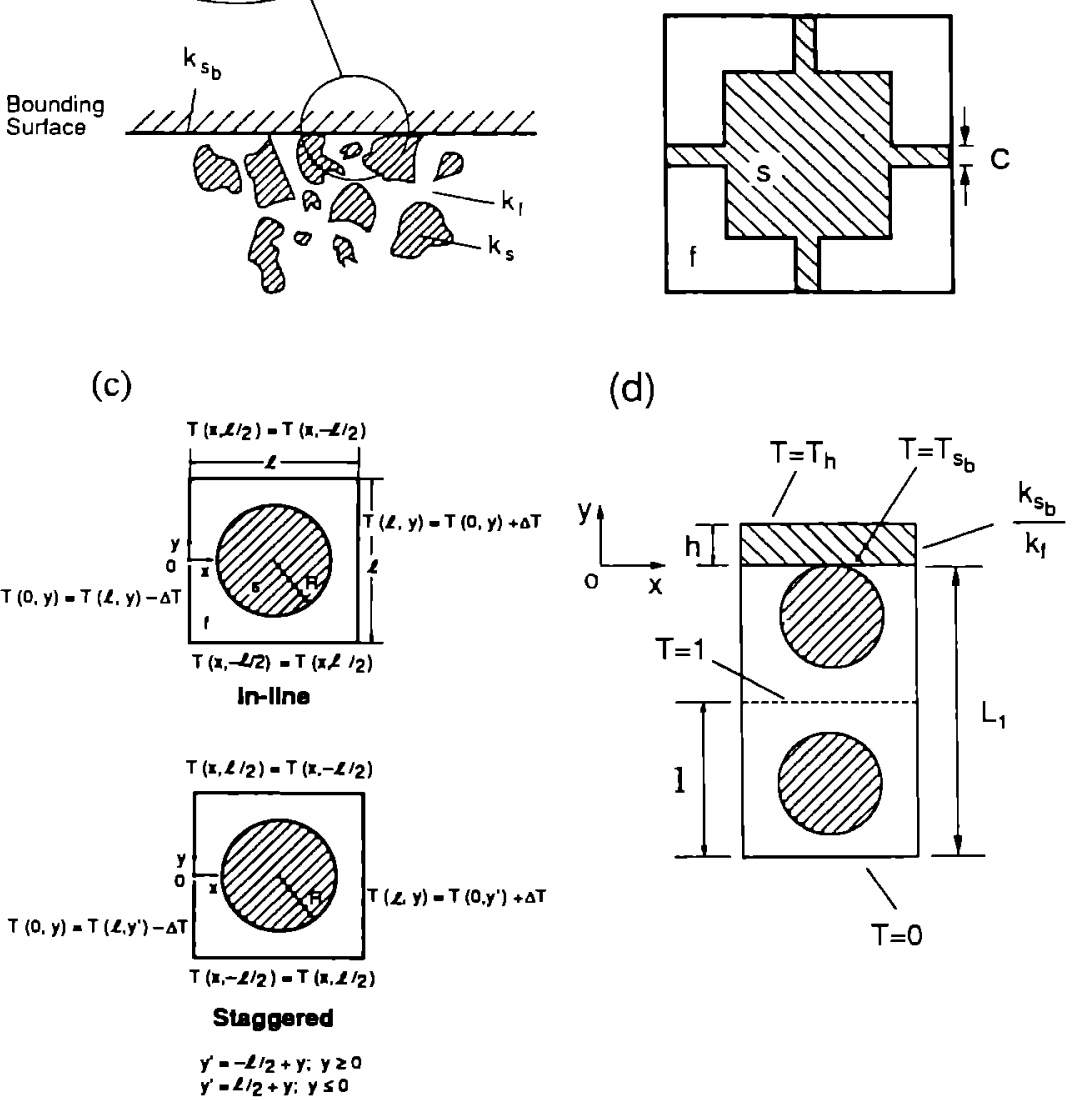

Fig. I. (a) Rendering of the phase distributions near a solid bounding surface. (b) two-dimensional unitcell models for nonconnected and interconnected square cylinders, (c) the periodic temperature boundary conditions used for the computation of the bulk effective conductivity for a circular cylinder, and (d) the

computational domain used for the study of the slip and no-slip temperature boundary conditions.

whether the plain medium is the fluid or another solid. When the temperature slip is allowed, a constant $k_{\mathrm{c} 1}=k_{\mathrm{c}}$ is used for $-\infty<y<0$.

As mentioned earlier, a slip at the interface occurs when the temperature at the interface is found by the extrapolation of the temperature distribution in the bulk of the porous medium. In order to model this slip, we construct a temperature slip boundary condition similar to that of Beavers and Joseph [5] for the hydrodynamic boundary condition at the porous- plain media interface. The temperature slip boundary condition is given by

$$
\left.\frac{\mathrm{d}\langle T\rangle_{Y}}{\mathrm{~d} y^{\prime}}\right|_{y=0^{-}}=\frac{\alpha_{T}}{\lambda}\left(T^{-}-T^{+}\right)
$$

where $\alpha_{T}$ is the dimensionless slip coefficient, $T^{-}$the porous medium interfacial temperature, $T^{+}$the plain medium interfacial temperature, and $\lambda$ a pore-level length scale. The length scale $\lambda$ is expected to be the 
order of the unit-cell length. Here we will use $\lambda=l$ where $f$ is the average linear dimension of the unit cell. In equation (2) the temperature gradient is cvaluated on the porous medium side, however, it can also be written in terms of the temperature gradient on the plain medium side, but $\alpha_{T}$ will be different. Using the bulk $k_{\mathrm{c}}$ and if the plain medium is the fluid, the continuity of the heat flux gives

$$
\left.k_{\mathrm{c}} \frac{\mathrm{d}\langle T\rangle_{\mathrm{r}}}{\mathrm{d} y}\right|_{y=0^{-}}=\left.k_{\mathrm{r}} \frac{\mathrm{d} T}{\mathrm{~d} y}\right|_{y=0^{-}}
$$

which is the second boundary condition that has to be used along with the slip boundary condition. Using this continuity of heat flux, equation (2) can then be written in terms of the plain medium temperature gradient. Note that in the hydrodynamic problem the condition given by equation (3) for the shear stress is not satisfied. This is because generally the Darcy law is used for the porous medium, and therefore, the boundary shear stress in the porous medium is generally neglected.

Prat $[6,7]$ considers heat conduction adjacent to the bounding surface of a two-dimensional porous medium. He describes [6] the different levels of description (microscopic and macroscopic) of the boundary condition for the Dirichlet- or the Neumann-type boundary conditions. He uses a periodic structure made of square cylinders. For this model he solves for the energy and the b equations (b equations will be defined in the next section). He reports a negligible difference in the interfacial temperature obtained using the macroscopic or the volume-averaged microscopic (local) model. By an order of magnitude analysis, Prat shows that the error introduced by using the macroscopic boundary condition is of the order of the temperalure deviation about the mean (i.e. $T^{\prime}=T-\langle T\rangle_{1}$ ). This error is larger for the Neumann boundary condition and increases as $k_{\mathrm{s}} / k_{\mathrm{r}}$ increases. Prat [7] extends his interfacial examination to a plain fluid layer bounding the porous medium. He considers two macroscopic models, one having a constant $k_{\mathrm{c} \perp}$ and the other a variable $k_{\mathrm{c} \perp}$. Again, he finds the difference to be small. As will be shown, this error is more noticeable when the bounding medium is not the fluid saturating the porous medium. For the variable effective conduclivity model, Prat [7] calculates the local transverse conductivity using equation (1). To find $k_{\mathrm{c} \perp}(y)$, he assumes a linear variation of the area-averaged temperature to obtain the volume-averaged temperature $\langle T\rangle_{1}$. Therefore, his calculated $k_{\mathrm{c} \perp}(y)$ depends on several parameters such as $k_{\mathrm{s}} / k_{\mathrm{r}}$, the width $L_{\mathrm{l}}$ of the porous medium, and the size of the averaging volume. However, we expect $k_{\mathrm{r} \perp}(y)$ to depend only on the local parameters and this will be examined further.

Since the bulk effective conductivity is used in the temperature slip model, we now examine the existing treatments of the bulk $k_{\mathrm{c}}$. The problem of heat con- duction through a fully salurated porous medium has been extensively examined bolh experimentally and theoretically. Most of these studies have focused on the bulk properties of the porous medium. The experiments are generally for packed beds of randomly arranged spherical particles. The theoretical invesligations are for a variety of unit-cell and phenomenological models. with porosity ranging from that for dilute suspensions to that for consolidated particles. A variety of particle-to-particle contacts have been assumed $[8,9]$. from point contact to compaction under gravitational and applied compacting forces. An extensive review of the literature on conduction in packed beds, giving the available correlations, is provided in Kaviany [10].

Nozad et al. [11] conducted experiments with randomly packed beds of spheres. In these experiments the solid to fluid conductivity ratio $k_{\mathrm{s}} / k_{\mathrm{f}}$ spans a wide range by using different solid (aluminum, bronze, stainless-steel and urea-formaldehyde) and fluid (air, glycerol and water) pairs. The effective conductivity is also calculated using the finite-difference method for a periodic structure of in-line nonconnecled square cylinders, as shown in Fig. l(b). Their numerical results show that for high $k_{\mathrm{s}} / k_{\mathrm{r}}$ the effective conductivity reaches an asymptote. However, the experiments for randomly close packed beds of spheres show that the effective conductivity increases monotonically with $k_{\mathrm{s}} / k_{\mathrm{f}}$. This is due to the contacts occurring between the spheres. Then in order to simulate the packed beds of spheres, Nozad et al. allow for contact between the particles by using connecling arms (or contact areas) of thickness $c$ having the same conductivity as the solid as shown in Fig. l(b). With this geometry the solid phase becomes continuous (while the fluid phase becomes discontinuous). The thickness of the contact area is found by malching the computed effective conductivity for $\varepsilon=0.36$ to the experimental results for $0.39 \leqslant \varepsilon \leqslant 0.41$. Nozad et al. report that $c / a=0.02$ gives the best fit to the experimental results. In a later communication by Shonnard and Whitaker [12] an error in the Nozad $e$ c al. analysis is acknowledged and the ratio $c / a$ is changed to 0.01 . As we will show below, even $c / a=0.01$ overestimates the experimental results by $300 \%$. This will be discussed further in Section 4.2.

The problem of heat conduction in a bed of nonconsolidated particles is also studied by Saez et al. [13] for a three-dimensional medium made of cubic particles with $k_{s}=0$. They study the effect of structure anisotropy by considering rectangular cross sections. They report that for isotropic structures the effective conductivity depends only on porosity and the threedimensional effects are not very important.

In this study the effective conductivity for periodic arrangements of circular and square cylinders is determined using the volume-averaging technique and the pointwise solution to the conduction equation. For the bulk effective conductivity $k_{\mathrm{c}}$ the effects of the porosity $\varepsilon$, the arrangement, the particle shape, and 
$k_{\mathrm{s}} / k_{\mathrm{i}}$, on $k_{\mathrm{c}}$ are examined. Both the no-slip temperature boundary condition, using a variable effective conduclivity, and the slip condition, are examined. The effects of $\varepsilon, k_{\mathrm{s}} / k_{\mathrm{f}}, k_{\mathrm{s}_{\mathrm{f}}} / k_{\mathrm{f}}$, and the phase distributions near the bounding surface, on $k_{\mathrm{c} \mid}(y)$ and $k_{\mathrm{c} \perp}(\mathrm{l})$ are examined.

\section{LOCAL VOLUME AVERAGING}

In the following we briefly review the existing theoretical treatment of the effective conductivity tensor by Carbonell and Whitaker [14]. Starting from the energy equation for the solid and the stagnant fluid

$$
\nabla \cdot k_{s} \nabla T_{s}=0 \text { and } \nabla \cdot k_{\mathrm{r}} \nabla T_{\mathrm{r}}=0
$$

with the boundary conditions on $A_{\text {rs }}$

$$
\mathbf{n}_{\mathrm{fs}_{\mathrm{s}}} \cdot k_{\mathrm{f}} \nabla T_{\mathrm{f}}=\mathbf{n}_{\mathrm{ss}} \cdot k_{\mathrm{s}} \nabla T_{\mathrm{s}} \text { and } T_{\mathrm{r}}=T_{\mathrm{s}} .
$$

By using the theory of local volume averaging and under the assumption of local thermal equilibrium [15]. a single heat conduction equation is written as

$$
\nabla \cdot\left(\mathbf{K}_{\mathrm{c}} \cdot \nabla\langle T\rangle_{\mathrm{v}}\right)=0
$$

where $\mathbf{K}_{\mathrm{c}}$ is the effective conductivity tensor. The local volume-averaged temperature is defined as

$$
\langle T\rangle_{1}=\frac{1}{V} \int_{1} T \mathrm{~d} V=\varepsilon_{\mathrm{s}}\langle T\rangle_{\mathrm{i}}+\varepsilon_{\mathrm{r}}\langle T\rangle_{\mathrm{r}}
$$

The effective conductivity tensor is defined through the volume-averaged energy equation which contains the local deviation $T_{\mathrm{r}}^{\prime}$

$\nabla \cdot\left\{\left[\varepsilon k_{\mathrm{r}}+\left(1-\varepsilon_{\mathrm{r}}\right) k_{\mathrm{s}}\right] \nabla\langle T\rangle_{\mathrm{r}}+\frac{k_{\ulcorner}-k_{\mathrm{s}}}{V} \int_{\mathrm{Al}_{\mathrm{rs}}} n_{\mathrm{rs}} T_{\mathrm{r}}^{\prime} \mathrm{d} A\right\}=0$

where

$$
T_{\mathrm{r}}^{\prime}=T-\langle T\rangle_{r}^{r} .
$$

In order to relate the local temperature deviations to the gradient of the volume-averaged temperature, the following transformations are introduced :

$$
T_{\Gamma}^{\prime}=\mathbf{b}_{\Gamma} \cdot \nabla\langle T\rangle_{1} \text { and } T_{s}^{\prime}=\mathbf{b}_{\mathrm{s}} \cdot \nabla\langle T\rangle_{\mathrm{r}} .
$$

Then the effective conductivity tensor is given by

$$
\mathbf{K}_{\mathrm{c}}=\left[\varepsilon k_{\ulcorner}+(1-\varepsilon) k_{\mathrm{s}}\right] \mathbf{I}+\frac{k_{\mathrm{f}}-k_{\mathrm{s}}}{V} \int_{\mathrm{A}_{\mathrm{f}}} \mathbf{n}_{\mathrm{fs}} \mathbf{b}_{\mathrm{f}} \mathrm{d} A .
$$

The $\mathbf{b}_{\mathrm{s}}$ and $\mathbf{b}_{\mathrm{f}}$ vectors are found by solving the $\mathbf{b}$ equations given by

$$
\nabla^{2} \mathbf{b}_{\mathrm{r}}=0 \text { and } \nabla^{2} \mathbf{b}_{\mathrm{s}}=0
$$

with the boundary condition on $A_{\mathrm{fs}}$

$$
k_{\mathrm{f}} \mathbf{n}_{\mathrm{fs}} \cdot \nabla \mathbf{b}_{\mathrm{f}}=k_{\mathrm{s}} \mathbf{n}_{\mathrm{fs}} \cdot \nabla \mathbf{b}_{\mathrm{s}}+\mathbf{n}_{\mathrm{rs}}\left(k_{\mathrm{s}}-k_{\mathrm{f}}\right) \text { and } \mathbf{b}_{\mathrm{f}}=\mathbf{b}_{\mathrm{s}} \text {. }
$$

The periodic boundary conditions are given by

$$
\mathbf{b}_{\mathrm{f}}\left(\mathbf{x}+\ell_{\mathrm{i}}\right)=\mathbf{b}_{\mathrm{r}}(\mathbf{x}) \text { and } \mathbf{b}_{\mathrm{s}}\left(\mathbf{x}+\ell_{i}\right)=\mathbf{b}_{\mathrm{s}}(\mathbf{x})
$$

where /, is the periodicity vector.

\section{SOLUTION METHOD}

The two-dimensional steady-state heat conduction equation is solved for a periodic structure of circular and square cylinders using the finite-difference approximations. The unit cells used in the computations are shown in Figs 1 (b) and (c). For the square cylinder shown in Fig. I(b) the porosity, including the areas of the connecting arms, is given by

$$
\varepsilon=1-\left[\frac{a^{2}}{r^{2}}+\frac{2(r-a) c^{\prime}}{r^{2}}\right]
$$

and for the circular cylinder shown in Fig. I(c) the porosity is

$$
\varepsilon=1-\frac{\pi d^{2}}{4} \frac{r^{2}}{}
$$

For a unit cell having a circular cylinder, a cylindrical-Cartesian grid overlaying scheme along with a bilinear interpolation are used to communicate between the two coordinates [1]. The governing equation in the Cartesian coordinates is

$$
\hat{\partial}\left(\begin{array}{c}
\hat{c} T \\
\hat{\partial} \mathrm{x}
\end{array}\right)+\frac{\hat{c}}{\partial \mathrm{y}^{\prime}}\left(\begin{array}{c}
\partial T \\
k \\
\partial \mathrm{y}^{\prime}
\end{array}\right)=0
$$

and for the cylindrical coordinates we have

$$
\frac{1}{r} \frac{\hat{c}}{\partial r}\left(k r \frac{\partial T}{\hat{\partial} r}\right)+\frac{1}{r 2} \frac{\hat{c}}{\hat{\partial} \theta}\left(k \frac{\hat{c} T}{\partial \theta}\right)=0
$$

These equations are nondimensionalized using $/$ as the length scale, and $\Delta T$ for the temperature. This temperature difference $\Delta T$ is the change in temperature that occurs over one cell and away from the interface. In the subsequent sections all the temperatures and lengths are dimensionless, unless otherwise specified. Variable conductivities are assumed in equations (17) and (18) in order to accommodate the step variation in the conductivity across the solidfluid interface (instead of using the continuity of heat flux given by equation (5)). This step variation in conductivity is modeled using the harmonic mean given by Patankar [16].

When a circular cylinder is used, iterations are performed alternatively in the two grid nets, and the values of the unknown at the boundaries of these grid nets are obtained using the bilinear interpolation. This bilinear interpolation is performed in the overlaying region, which extends 3-4 grid nodes. An unknown temperature on the boundary of the cylindrical grid net is obtained by the bilinear interpolation, using the four surrounding nodes in the Cartesian grid. The same procedure is used for the lemperature on the boundary of the Cartesian grid net. In order to solve for the temperature field, five iterations are carried out 
in the Cartesian domain. The bilinear interpolation is then used to find the boundary conditions for the cylindrical domain, where the next five iterations are performed. After these iterations, interpolations are made back to the Cartesian grid (for every cylinder in the domain).

The applied boundary conditions differ depending on the region examined (i.e. the bulk or the interfacial region). When a unit cell is used to compute the bulk effective conductivity of the packed bed, the boundary conditions used are those given in Fig. I(c) (for inline and staggered arrangements of circular cylinders). Periodic boundary conditions are used in both directions. For the interfacial region, shown in Fig. 1(d), the periodic boundary condition given by

$$
T\left(0, y^{\prime}\right)=T(\kappa, y)
$$

is used in the $x$-direction. In the $y$-direction prescribed temperatures

$$
T(x, h)=T_{h} \text { and } T\left(x,-L_{1}\right)=0
$$

are used at the upper and lower boundaries. Figure I(d) shows also the condition

$$
T\left(x, 1-L_{1}\right)=1
$$

resulting from the use of the temperature difference $\Delta T$ across the lower cell for the normalization. In practice, for a given phase distribution near the surface, $T_{h}$ is adjusted such that the equality given by equation $(21)$ is satisfied. Note that since $\langle T\rangle_{A_{1}}(\mathrm{y}=$ $\left.-L_{1}\right)=0$ we have

$$
\begin{aligned}
\Delta T=\left[\langle T\rangle_{A_{1}}\left(y^{\prime}=1-L_{1}\right)\right. & \left.-\langle T\rangle_{A_{1}}\left(y=-L_{1}\right)\right] \\
= & \langle T\rangle_{A_{\mathrm{T}}}\left(y=1-L_{1}\right)
\end{aligned}
$$

where we now have used $\langle T\rangle_{A_{1}}$ which is the areaaveraged temperature in the $x$-direction and is defined as

$$
\langle T\rangle_{\lambda_{1}}(x, y)=\int_{-0.5}^{0.5} T\left(x+x^{\prime}, y\right) \mathrm{d} x^{\prime} .
$$

Since the periodic boundary conditions are used in the $x$-direction $\langle T\rangle_{A_{1}}(x, y)$ reduces to $\langle T\rangle_{A_{\mathrm{r}}}(y)$. A similar area average in the $y$-direction is defined by

$$
\langle T\rangle_{A_{1}}(x, y)=\int_{-0.5}^{0.5} T\left(x, y+y^{\prime}\right) \mathrm{d} y^{\prime} .
$$

The effective conductivity for a unit cell can be calculated using equation (11), requiring the solutions to the b equations (12). However, the effective conductivity can also be computed directly by using the temperature field. This is preferred because the b equations and their boundary conditions are more complicated than the energy equation. By taking the volume average of the pointwise temperature field defined by

$$
\langle T\rangle_{v}(x, y)=\int_{-0.5}^{0.5} \int_{-0.5}^{0.5} T\left(x+x^{\prime}, y+y^{\prime}\right) \mathrm{d} x^{\prime} \mathrm{d} y^{\prime}
$$

the effective conductivity tensor becomes

$$
\langle\mathbf{q}\rangle_{\mathrm{r}}=-\frac{\mathbf{K}_{\mathrm{c}}}{k_{\mathrm{r}}} \cdot \nabla\langle T\rangle_{\mathrm{r}} .
$$

The volume-averaged heat flux vector is

$$
\langle\mathbf{q}\rangle_{\mathrm{r}}=-\left\langle\frac{k}{k_{\mathrm{f}}} \nabla T\right\rangle_{\mathrm{l}}
$$

For the unit-cell models shown in Figs. I(b) and (c) the area-averaged $x$-direction heat flux, with average taken over $y$ is independent of $x$, i.e.

$$
\left\langle q_{x}\right\rangle_{A_{1}}(x)=\int_{-0.5}^{0.5}-\frac{k}{k_{\mathrm{f}}} \frac{\partial T\left(x, y^{\prime}\right)}{\partial x} \mathrm{~d} y^{\prime}=\left\langle q_{x}\right\rangle_{A_{1}}(x=0) .
$$

This is because the periodic (or adiabatic) boundary condition is used in the $y$-direction. From equation (28), we oblain the volume-averaged $x$-component of the heat flux vector (equation (27)) to be

$$
\left\langle q_{x}\right\rangle_{1}=\left\langle q_{x}\right\rangle_{A_{x}}(x)=\left\langle q_{x}\right\rangle_{\lambda_{1}}(x=0) \text {. }
$$

The gradient of the volume-averaged temperature is the temperature gradient imposed as the boundary condition (i.e. dimensionless $\Delta T$ ). Then, the effective conductivity becomes

$$
\frac{k_{\mathrm{e}}}{k_{\mathrm{r}}}=\frac{\left\langle q_{x}\right\rangle_{A_{\mathrm{r}}}(x=0)}{\Delta T} .
$$

We have compared the bulk effective conductivity calculated using equation (30) and the one using the solution to the $\mathbf{b}$ equations for a periodic structure of square cylinders, and an agreement of less than $0.1 \%$ is obtained. All the results presented below are computed using the pointwise temperature field.

\section{BULK EFFECTIVE CONDUCTIVITY}

In this section, the bulk effective conductivity for a unit cell is computed using the unit cells shown in Figs. 1(b) and (c). In these computations the effects of $k_{\mathrm{s}} / k_{\digamma}$, porosity, particle geometry, and arrangement, on the effective conductivity are examined.

\subsection{Nonconnected particles}

We will first examine nonconnected particles (i.e. discontinuous solid phase), where an asymptotic behavior for $k_{\mathrm{e}} / k_{\mathrm{r}}$ is found for $k_{\mathrm{s}} / k_{\mathrm{f}} \rightarrow \infty$.

4.1.1. Arrangement. The effect of the particle arrangement on the bulk effective conductivity is examined using both the in-line and the staggered arrangements of circular cylinders. In the staggered arrangement, compared to the in-line arrangement, the two adjacent columns are dislocated up or down by one half of a unit-cell size.

The effect of the particle arrangement on $k_{\mathrm{c}} / k_{\mathrm{r}}$, for several porosities, is shown in Fig. 2(a) for circular cylindrical particles. As expected, for high porosities (i.e. $\varepsilon \geqslant 0.8$ ) the effective conductivity does not 
depend on the arrangement. In this range of porosity the scaled particle diameter is smaller and the influence of the neighboring particles becomes less significant. As the porosity decreases, this interaction becomes more important and the difference between the effective conductivity of the two arrangements increases. Figure 2(a) shows that the in-line arrangement results in a larger effective conductivity. This is because in the in-line arrangement adjacent cylinders are closer than in the staggered arrangement. For the lowest porosity used in this study $(\varepsilon=0.4)$, the difference in the effective conductivity, between the staggered and the in-line arrangements, is about $18 \%$.

4.1.2. Particle geometry. The effect of the particle shape on the effective conductivity is examined by using circular and square cylinders and the results are shown in Fig. 2(b). As with the particle arrangement, at high porosities the particle shape did not influence the effective conductivity. However, as the porosity decreases the effect of the particle shape becomes significant. For porosities larger than 0.5 , the effective conductivity for a bed made of square cylinders is larger than that for circular cylinders. Away from the center of the cylinder, the distance (in the heat flux direction) between two adjacent circular cylinders increases. This increased distance represents extra resistance $\left(k_{\mathrm{s}} / k_{\mathrm{f}}>1\right)$ to the heat flow and the effective conductivity of the medium decreases. For porosities less than 0.5 , the circular cylinders have a higher effective conductivity than the square cylinders. This is because for the same porosity, near the center of the cell the surfaces of two adjacent circular cylinders are closer than the square cylinders. As the distance between adjacent cylinders becomes smaller (i.e. lower porosity), the heat flux through the center of the particle becomes more significant compared to that away from the center. Thus, the effective conductivity of the circular cylinders becomes larger.

4.1.3. Correlations. In order to provide a correlation between the effective conductivity of a bed of nonlouching particles and the bed parameters ( $\varepsilon$ and $k_{\mathrm{s}} / k_{\mathrm{f}}$ ) curve fits are made through the numerical results presented in Figs. 2(a) and (b). As a theoretical guide, we consider a simple model for the nontouching square cylinders. In this model, we assume a one-dimensional heat conduction through a composite slab having a parallel and series arrangement of resistances. We apply the equivalent thermal circuit and obtain the effective conductivity of the composite slab. For the nonconnected square cylinder shown in Fig. $1(\mathrm{~b}), k_{\mathrm{c}} / k_{\mathrm{r}}$ is written in terms of $\varepsilon$ and $k_{\mathrm{s}} / k_{\mathrm{r}}$ as

$$
\frac{k_{\mathrm{e}}}{k_{\mathrm{f}}}=\frac{\frac{k_{\mathrm{s}}}{k_{\mathrm{f}}} \phi^{1 / 2}+\left(1-\phi^{1,2}\right)}{\frac{k_{\mathrm{s}}}{k_{\mathrm{f}}} \phi^{1 / 2}\left(1-\phi^{1,2}\right)+\left(1-\phi^{1 / 2}\right)^{2}+\phi^{1 / 2}}
$$

(a)

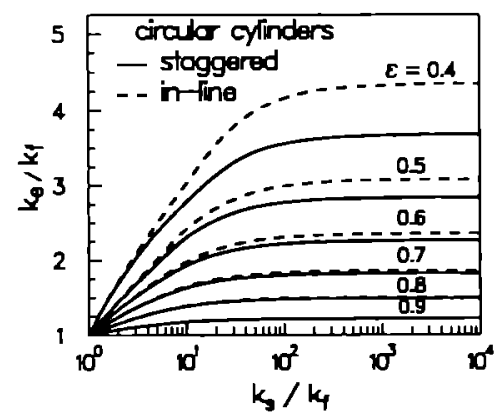

(b)

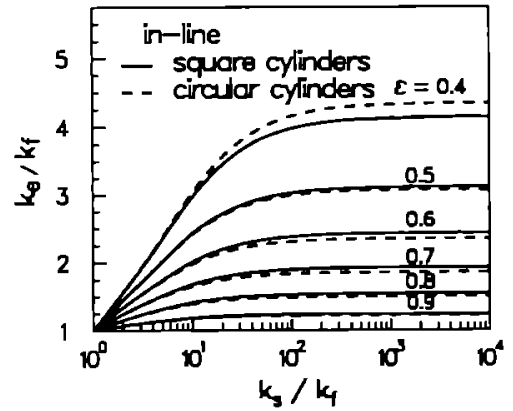

(c)

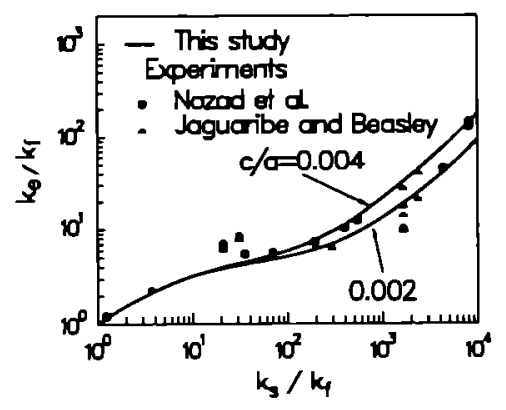

FIG. 2. (a) Effect of the particle arrangement on $k_{\mathrm{e}} / k_{\mathrm{f}}=\left(k_{\mathrm{c}} / k_{\mathrm{f}}\right)\left(k_{\mathrm{s}} / k_{\mathrm{f}}\right)$ using in-line and staggered arrangements of circular cylinders, (b) effect of the particle geometry on $k_{\mathrm{e}} / k_{\mathrm{f}}=\left(k_{\mathrm{e}} / k_{\mathrm{f}}\right)\left(k_{\mathrm{s}} / k_{\mathrm{f}}\right)$ using circular and square cylinders, and (c) comparison of $k_{\mathrm{c}} / k_{\mathrm{r}}=\left(k_{\mathrm{c}} / k_{\mathrm{r}}\right)\left(k_{\mathrm{s}} / k_{\mathrm{f}}\right)$ from the numerical results for interconnected square cylinders with the available experimental results for packed beds of spheres. 
Table I. Correlations for function $f(\phi)$, used for the prediction of the bulk $k_{\mathrm{c}}$ for $0.1 \leqslant \phi \leqslant 0.6$

\begin{tabular}{lc}
\hline $\begin{array}{c}\text { Geometry or } \\
\text { arrangement }\end{array}$ & Expression \\
\hline $\begin{array}{c}\text { In-line circular } \\
\text { cylinders }\end{array}$ & $f(\phi)=0.88-0.38 \phi+0.93 \phi^{2}$ \\
$\begin{array}{c}\text { Staggered } \\
\text { circular } \\
\text { cylinders }\end{array}$ & $f(\phi)=-0.2 \mathrm{I}+2.77 \phi-6.77 \phi^{2}+10.82 \phi^{3}$ \\
$\begin{array}{c}\text { In-line square } \\
\text { cylinders }\end{array}$ & $f(\phi)=0.83+0.18 \phi$ \\
\hline
\end{tabular}

where $\phi$ is the volume fraction of the solid phase (i.e. $\phi=1-\varepsilon$ ). For large $k_{\mathrm{s}} / k_{\mathrm{f}}$, the numerical results in Figs. 2(a) and (b) approach the expected asymptotes. These asymptotes are also obtained from this circuit model for $k_{\mathrm{s}} / k_{\mathrm{f}} \rightarrow \infty$, and the result is

$$
\frac{k_{\mathrm{c}}}{k_{\mathrm{r}}}\left(\frac{k_{\mathrm{s}}}{k_{\mathrm{f}}} \rightarrow \infty\right)=\frac{\mathrm{l}}{1-\phi^{1 ; 2}} .
$$

The model assumes that the heat conduction is onedimensional in each component of the slab. However, the heat conduction is two-dimensional, especially for large $k_{\mathrm{s}} / k_{\mathrm{f}}$. In order to include these two-dimensional effects, a coefficient $f(\phi)$ is introduced in the numerator of equation (31). This coefficient is introduced to match the asymptotes at large $k_{\mathrm{s}} / k_{\mathrm{r}}$. Then, the effective conductivily given by equation (31) becomes

$$
\begin{aligned}
\frac{k_{\mathrm{c}}}{k_{\mathrm{r}}} & =g\left(\phi \cdot \frac{k_{\mathrm{s}}}{k_{\mathrm{f}}}\right) \\
& =\frac{f(\phi) \frac{k_{\mathrm{s}}}{k_{\mathrm{f}}} \phi^{1 / 2}+\left(1-\phi^{1 / 2}\right)+[1-f(\phi)] \phi^{1 / 2}}{\frac{k_{\mathrm{s}}}{k_{\mathrm{r}}} \phi^{1 / 2}\left(1-\phi^{1 / 2}\right)+\left(1-\phi^{1 / 2}\right)^{2}+\phi^{1 / 2}} .
\end{aligned}
$$

The third term in the numerator is introduced to satisfy the limit $k_{\mathrm{c}} / k_{\mathrm{f}}=1$, when $k_{\mathrm{s}} / k_{\mathrm{\digamma}}=1$. By taking the limit of equation (33) at large $k_{\mathrm{s}} / k_{\mathrm{f}}$, we find that $f(\phi)$ is given by

$$
f(\phi)=\left(1-\phi^{1 / 2}\right) \frac{k_{\mathrm{c}}}{k_{\mathrm{r}}}\left(\frac{k_{\mathrm{s}}}{k_{\mathrm{r}}} \rightarrow \infty\right) .
$$

The curve fits for the function $f(\phi)$ are found using the value obtained from equation (34) and the numerical results for $\left(k_{\mathrm{c}} / k_{\mathrm{f}}\right)\left(k_{\mathrm{s}} / k_{\mathrm{f}}=10^{4}\right)$. These functions are given in Table 1 . The expressions for $k_{\mathrm{c}} / k_{\mathrm{r}}$ are in very good agreement with the numerical results especially for large $k_{\mathrm{s}} / k_{\mathrm{r}}$ (as expected). The error using the expressions is about $0.5 \%$ for large $k_{\mathrm{s}} / k_{\mathrm{f}}\left(\geqslant 10^{3}\right)$ for circular cylinders, and it is about $4 \%$ for $k_{\mathrm{s}} / k_{\mathrm{r}}=5$ (for the in-line arrangement, and $\varepsilon=0.9$ ).

\subsection{Interconnected particles}

When solving b equations (12)-(14), Nozad et al. [11] encounter numerical instabilities for $k_{\mathrm{s}} / k_{\mathrm{r}}>100$. They use a perturbation method with $\left(k_{\mathrm{s}} / k_{\mathrm{r}}\right)^{-1}$ as the perturbation parameter to remedy this problem. They solve the $b$ equations for the various orders of the perturbation. The effective conductivity is computed using equation (1I). In the next step of the derivation, by taking the limit when $k_{\mathrm{s}} / k_{\mathrm{r}} \rightarrow \infty$, the integral containing the first-order term of the expansion was dropped from equation (5.1-2) in Nozad [17] and equation (3.23) in Nozad et al. [11]. This produces an overestimation of the contact area. This overestimation can be shown by replacing the interconnected square cylinder shown in Fig. I(b) by a slab placed in the center parallel to the direction of the heat flow and having a uniform thickness $c$ (same as before). In this geometry, the heat conduction is onedimensional and the heat flux is less than the corresponding two-dimensional heat flow for the interconnected geometry shown in Fig. 1(b). The prediction of Nozad et al. is lower than this onedimensional case. As was mentioned earlier, this error was later noted [12] and a $c / a=0.01$ was suggested. However, our results (Fig. 2(c)) show that this thickness overpredicts the effective conductivity for a packed bed of spheres. To validate our results for $c / a=0.0$ I we use an approximation where the temperature of the square cylinder is constant for very large $k_{\mathrm{s}} / k_{\mathrm{f}}\left(\geqslant 10^{3}\right)$. This assumption of constant temperature for the square cylinder is verified by our numerical results. For very large $k_{s} / k_{\mathrm{r}}$, the heat flowing through the thickness $c / a$ constitutes nearly all the heat flowing through the cell. Using the one-dimensional conduction for the arm length $1-a / f$, thickness $c / a, k_{\mathrm{s}} / k_{r}$, and the temperature difference $(\Delta T=1)$, the effective conductivity can be estimated from equation (30). This approximation gives an effective conductivity, for $\varepsilon=0.36, c / a=0.01$, and $k_{\mathrm{s}} / k_{\mathrm{r}}=10^{4}$ which is higher by $20 \%$ compared to our numerical results and higher by $300 \%$ compared to that of Shonnard and Whitaker [12].

In Fig. 2(c) we show the results for two ratios of $c / a(0.002$ and 0.004$)$ in order to cover some of the scatter in the experimental data at high $k_{\mathrm{s}} / k_{\mathrm{r}}$. As is shown, a $c / a=0.002$ is more appropriate for representing the experimental results. The experimental results presented in Fig. 2(c) were obtained by Nozad et al. [11] and Jaguaribe and Beasley [18]. Nozad et al. perform the experiments for a packed bed of spheres using different solid (aluminum, bronze, stainless steel and urea-formaldehyde) and fluid (air, glycerol and water) pairs. In the experiments conducted by Jaguaribe and Beasley the packed beds are made of glass spheres and steel balls. The fluids used in these experiments are helium, carbon dioxide, water and air.

\section{NEAR BOUNDING SURFACES}

In this section, we study the anisotropy in the phase distributions, and therefore, in $\mathbf{K}_{\mathrm{e}} / k_{\mathrm{f}}$ near the interface of a porous medium and a bounding medium which can be either the fluid saturating the porous medium 
or a solid. Near the interface, the local porosity (in an areal sense) changes and consequently the local effective conductivity changes. Using the pointwise solution to $T(x, y)$ the variation of $\mathbf{K}_{\mathbf{c}}\left(y^{y}\right) / k_{\mathrm{r}}$ is examined using the local volume-averaging technique. The slip boundary condition is also examined using the local volume-averaging technique. The size of the averaging volume is varied with the position such that a large averaging volume is used in the bulk region of the porous medium, while an infinitesimal volume is used at the interface. More detailed discussion about the averaging volume can be found in our treatment of the hydrodynamics near the interface [12]. The averaging volume used at the interface is the small grid size used in the numerical integration, and a unitcell size for $y \leqslant-1 / 2$, and $-2 y$ for any point in between. The volume-averaged temperature for $0 \geqslant y \geqslant-1 / 2$ is

$$
\langle T\rangle_{y} \cdot(x, y)=\frac{-1}{2 y} \int_{2 y}^{0} \int_{-0.5}^{0.5} T\left(x+x^{\prime}, y^{\prime}\right) \mathrm{d} x^{\prime} \mathrm{d} y^{\prime} .
$$

Since a variable averaging volume is used here, the derivation of the averaged energy equation has to be modified. This modification arises from a change in the volume averaging theorem when accounting for the variable averaging volume. The averaging theorem is modified by Gray [19] and is given by

$$
\begin{aligned}
\langle\nabla T\rangle_{1}=\nabla\langle T\rangle_{1} & +\frac{1}{V} \int_{A_{1,}} T \mathbf{n} \mathrm{d} A \\
& +\frac{\nabla V}{V}\left(\langle T\rangle_{1}-\frac{1}{A_{V_{Y}}} \int_{A_{1}} T \mathrm{~d} A\right) .
\end{aligned}
$$

The terms between the parentheses are due to the variable averaging volume and vanish if the averaging volume is constant. Gray shows that if the condition

$$
\nabla V \cdot\left(\frac{\mathrm{l}}{A_{\mathrm{I}}} \int_{\mathrm{I}_{\mathrm{l}}} \frac{k}{k_{\mathrm{f}}} \nabla T \mathrm{~d} A-\frac{1}{V} \int_{V} \frac{k}{k_{\mathrm{f}}} \nabla T \mathrm{~d} V\right)=0
$$

is satisfied, then the averaged transport equation derived for a constant volume is applicable. For convection heat transfer, the convective flux term is also included in equation (37). Note that the $\nabla V$ vector only has a $y$-component, since the averaging volume does not vary in the $x$-direction. In the $y$-direction, the area- and volume-averaged $y$-direction heat flux are the same. This is due to the periodic boundary conditions used in the $x$-direction. Therefore, the term on the right-hand side of equation (37) is identically zero. Thus, using the averaged energy equation, i.e. equation (6), near the interface, is appropriate.

All the numerical results reported below are for circular cylindrical particles. The particles are nonconnected, and therefore, the solid phase is discontinuous.

\subsection{Slip boundary condition}

The slip boundary condition allows for the slip in the temperature at $\mathrm{y}^{\prime}=0$ and accounts for the local variation of the effeclive conductivily. The variation of the effective conductivity near the interface is lumped into the empirical slip coefficient $\alpha_{T}$. As mentioned in the introduction, the slip boundary condition has been previously used for heat Iransfer experiments in packed beds of spheres (e.g. Matsuura et al. [4]). In these experiments, the spheres in the packed bed are in direct contact with the bounding wall. Ideally, this problem is modeled by a porous medium having a prescribed temperature $T_{\mathrm{s}_{\mathrm{h}}}$ at the boundary. Then, the boundary condition given by equation (2) is replaced by

$$
\left.\frac{\mathrm{d}\langle T\rangle_{1}}{\mathrm{~d}^{\prime} \cdot}\right|_{y=0^{-}}=\alpha_{T}\left(T^{-}-T_{\mathrm{s}_{\mathrm{h}}}\right) .
$$

The temperature distribution for the one-dimensional heat flow with negligible radiation effects is found by solving

$$
\frac{\mathrm{d}^{2}\langle T\rangle_{1}}{\mathrm{~d} y^{2}}=0
$$

The boundary condition given by equation (38) is used at the interface $(y=0)$, along with

$$
\langle T\rangle_{V}(0)=T^{-} \text {and }\langle T\rangle_{Y}\left(-L_{1}\right)=0 .
$$

By solving equation (39) using the given boundary conditions, we have for $T$

$$
T^{-}=\frac{\alpha_{T} T_{\mathrm{s}_{\mathrm{h}}}}{\alpha_{T}-\frac{1}{L_{1}}}
$$

The model used to study this boundary condition is shown in Fig. 1(d). In our numerical simulations we assign a very large conductivity for the bounding solid $\left(k_{\mathrm{s}_{\mathrm{m}}} / k_{\mathrm{r}}=10^{4}\right)$ and this allows us to obtain a constant temperature $\left(T_{\mathrm{s}_{\mathrm{b}}}=T_{h}\right)$ at the nominal interface $(y=0)$.

The temperature slip coefficient $\alpha_{T}$ is calculated using equation (38), and the result is

$$
\alpha_{T}=\frac{\left.\frac{\mathrm{d}\langle T\rangle_{\mathrm{I}}}{\mathrm{d} y^{\prime}}\right|_{y=0^{-}}}{\left(T^{-}-T_{\mathrm{s}_{\mathrm{h}}}\right)} .
$$

The temperature $T^{-}$, shown in Fig. 3(a), is found by the extrapolation of the temperature distribution using the results away from the boundary $(y=-1)$. The temperature gradient in equation (42) is also calculated using the results away from the interface.

When the particle is in direct contact (point contact) with the bounding surface, the particle conductivity $k_{\mathrm{s}} / k_{\mathrm{r}}$ significantly influences the heat flux at the wall. 
Table 2. Effect of the particle conductivity on the slip coefficient for circular cylinders in contact with a solid bounding medium of high conductivity $\left(k_{-\mathrm{h}} / k_{\mathrm{f}}=10^{4}, \varepsilon=0.5\right)$

\begin{tabular}{|c|c|c|c|c|c|}
\hline$k \sqrt{ } / k_{\mathrm{r}}$ & $k_{\mathrm{v}} / k_{\mathrm{I}}$ & $\alpha_{T}$ & $T^{-}-T_{\mathrm{b}}$ & $q_{\text {crict }}$ & $q_{\mathrm{no}} \mathrm{Ni \textrm {r }}$ \\
\hline 5 & 2.01 & 7.11 & 0.14 & 2.01 & 1.72 \\
\hline 10 & 2.42 & 4.58 & 0.21 & 2.42 & 1.59 \\
\hline 50 & 2.92 & 2.90 & 0.34 & 2.92 & I. .23 \\
\hline
\end{tabular}

This is shown in Table 2, where the results show that the slip in the temperature increases as $k_{\mathrm{s}} / k_{\mathrm{f}}$ increases. In experiments for heal transfer in a packed bed of particles or a porous medium bounded by a solid wall the heat flux could be calculated by measuring two temperatures, onc is the wall temperature and the other is at the vicinity of the wall. Note that this approach does not allow for slip at the interface. Here we show the crror that could be caused by not allowing slip in computing the heat flux. The heat flux is calculated using the bulk effective conductivity, the temperature at the interface (i.e. $T_{s_{\mathrm{h}}}$ ) and the temperature one cell length away from the interface (i.e. $\left.\langle T\rangle_{1}\left(y^{\prime}=-1\right)\right)$. The no-slip heat flux is then given by

$$
q_{\text {noslip }}=\left[T_{s_{\mathrm{h}}}-\langle T\rangle_{\mathrm{V}}(y=-1)\right] \frac{k_{\mathrm{c}}}{k_{\mathrm{f}}}
$$

In Table $2 q_{\text {nos sip }}$ is compared to the heat flux obtained from the exact solution. The difference between the two heal fluxes increases with the solid conductivity and a difference of $60 \%$ is obtained for $k_{\mathrm{s}} / k_{\mathrm{r}}=50$.

An altempt is made to find a correlation between the slip coefficient $x_{T}$ and the bed parameters $\varepsilon$ and $k_{\mathrm{c}} / k_{\mathrm{r}}$. We have found that these variables can be separated, i.e.

$$
\alpha_{\gamma_{7}}\left(k_{\mathrm{c}} / k_{\mathrm{r}}, \varepsilon\right)=\alpha_{T_{1}}\left(k_{\mathrm{r}} / k_{\mathrm{f}}\right) \alpha_{T_{2}}(\varepsilon) .
$$

Then, the correlation is found to be

$$
\begin{gathered}
\alpha_{T}=10.07\left(\frac{k_{\mathrm{e}}}{k_{\mathrm{r}}}\right)^{-2 .+1} \varepsilon^{-1.92} \\
\text { for } 0.5 \leqslant \varepsilon \leqslant 0.7 \text { and } \frac{k_{\mathrm{s}_{\mathrm{p}}}}{k_{\mathrm{r}}}=10^{4} .
\end{gathered}
$$

Figure 3(a) shows the distribution of the volumeand area-averaged temperalures for $k_{\mathrm{s}} / k_{\mathrm{r}}=50$ and $\varepsilon=0.5$. From this figure we notice that the boundary effect is limited to about half of the cylinder diameter. It also shows that the extrapolated temperature $T^{-}$at the interface is higher than the prescribed temperature of the interface. Note that in the available experimental results for packed beds of spherical particles, the extrapolated $T^{-}$is lower than the prescribed temperature. This is due to the different $\langle\varepsilon\rangle_{H}(y)$ near the boundary found for the periodic arrangement of circular cylinders and that of the random arrangement of spheres. For the packed bed of spheres, the porosity near the wall is higher than in the bulk and the local effective conductivity is lower. For this reason a higher temperature gradient exists near the wall. For the circular cylinders depicted in Fig. 1(d), the porosity near the wall is lower than in the bulk and this results in a higher local effective conductivity and a smaller temperature gradient near the interface. The packed bed of spheres can be simulated by moving the solid away from the surface tangent to the first layer of cylindrica! particles. When the interface is placed at a distance of $1 / 2$ away from the center of the particle, the slip in temperature is zero. When the interface is placed at a distance further than 1/2 a temperature slip similar to the one in the packed bed of spheres is oblained.

The slip boundary condition is also used for the case where the bounding medium is the fluid saturating the porous medium. In Fig. l(d) the solid wall is replaced by the fluid $\left(k_{s_{\mathrm{b}}} / k_{\mathrm{f}}=1\right)$. The results of the area- and volume-averaged lemperature for $k_{\mathrm{s}} / k_{\mathrm{f}}=50$ and $\varepsilon=0.5$ are shown in Fig. 3(b). The temperature $T^{-}$is extrapolated in the same way as done above for the solid bounding wall. From Fig. 3(b) we notice the boundary effect does not penetrate into the plain medium and $T^{+}$does not have to be extrapolated. The slip boundary condition is examined for different $\varepsilon$ and $k_{\mathrm{s}} / k_{\mathrm{f}}$. The slip coefficient was calculated using the same approach outlined when the particles are touching the bounding surface and the results of these calculations are given in Table 3 . The results for $\alpha_{T}$ are also shown in Fig. 3(c). These results show that $\alpha_{T}$ does not change significantly as the bulk porosity changes and is of the order of 10 for $k_{\mathrm{s}} / k_{\mathrm{r}}=5$. The slip in temperature occurring at the interface is also reported in Table 3 to indicate the error caused by the no-slip one-dimensional analysis. The difference between the heat fluxes evaluated using the local simulation and that using the no-slip one-dimensional model is also shown. The one-dimensional no-slip heat flux is calculated in the same way as the solid bounding wall using equation (43) with $T_{s_{\mathrm{b}}}$ replaced by $T^{+}$. The largest difference is about $9.5 \%$ and is for the lowest porosity $(\varepsilon=0.5)$.

The effect of the particle conductivity on the slip coefficient is also examined for $\varepsilon=0.5$. The results which are given in Table 4 show that over a wide range of $k_{\mathrm{s}} / k_{\mathrm{r}}$ the slip coefficient changes significantly. As the conductivity of the particle increases, the slip in temperature increases, because the local conductivity deviates more from the bulk effective conductivity due to the lower local porosity near the interface. For a given porosity and a variable bulk effective conductivity, the same behavior of $\alpha_{T}$ for a fluid bounding medium as that of the solid bounding medium is observed. Thus, we attempt to find a correlation similar to the one for the solid bounding medium given by equation (44). This relationship is given by

$$
\alpha_{T}=2.59 \frac{1}{\frac{k_{\mathrm{c}}}{k_{\mathrm{f}}}-1} \varepsilon^{-2.04}
$$


(c)

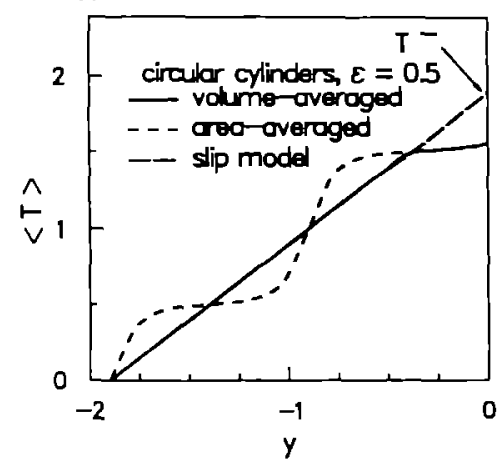

(c)

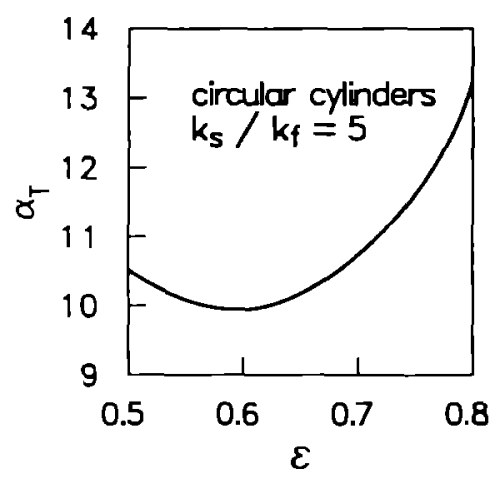

(b)

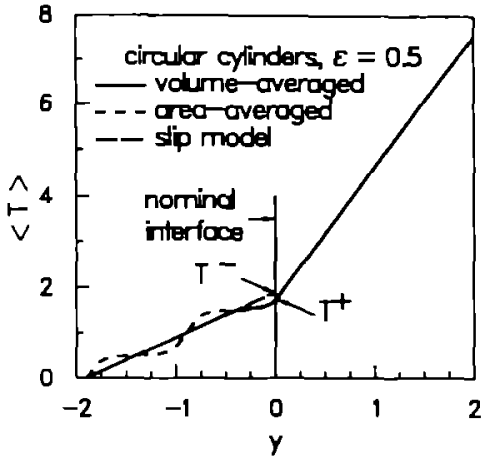

(d)

(e)
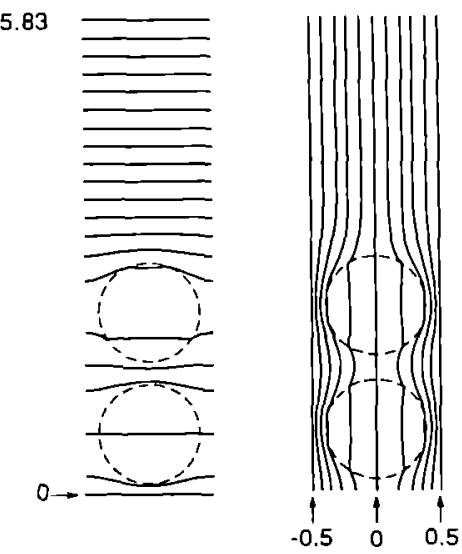

FiG. 3. (a). (b) Volume- and area-averaged temperature distributions for heal conduction in an in-line arrangement of circular cylinders $\left(\varepsilon=0.5, k_{\mathrm{b}} / k_{\mathrm{r}}=50\right)$ at the interface with (a) a near perfectly conducting solid bounding surface $\left(k_{\mathrm{s}_{\mathrm{h}}} / k_{\mathrm{r}}=10^{4}\right)$ and (b) the fluid bounding medium $\left(k_{\mathrm{r}} / k_{\mathrm{f}}=1\right)$, (c) variation of the slip coefficient with the porosity ror a packed bed of an in-line arrangement of circular cylinders $\left(k_{\mathrm{s}} / k_{\mathrm{r}}=5, k_{\mathrm{s}_{\mathrm{h}}} / k_{\mathrm{r}}=1\right)$, (d), (e) constant temperature contours for transverse (1'-direction) and longitudinal ( $x$-direction) heat flows, respectively $\left(k_{\sqrt{ }} / k_{\mathrm{r}}=5, k_{\sqrt{\mathrm{r}}} / k_{\mathrm{r}}=1\right)$.

$$
\text { for } 0.5 \leqslant \varepsilon \leqslant 0.8 \text { and } \frac{k_{s_{\mathrm{h}}}}{k_{\digamma}}=1 \text {. }
$$

\subsection{No-slip boundary condition}

The phase distributions near the interface are both anisotropic and nonuniform. Thus, the effective conductivity for this two-dimensional structure has two variable components. These are the longitudinal $k_{\mathrm{c}, 1}(y) / k_{\mathrm{r}}$ in the $x$-direction and the transverse component $k_{\mathrm{c} \perp}\left(y^{\prime}\right) / k_{\mathrm{r}}$ in the $y$-direction.

In order to determine $k_{\mathrm{e} \perp}(y) / k_{\mathrm{r}}$, we imposed a

Table 3. Efect of bulk porosity on the slip coefficient for an in-line arrangement of circular cylinders bounded by the fluid $\left(k_{\mathrm{s}_{\mathrm{p}}} / k_{\mathrm{f}}=1, k_{\mathrm{s}} / k_{\mathrm{r}}=5\right)$

\begin{tabular}{ccrccc}
\hline$\varepsilon$ & $k_{\mathrm{c}} / k_{\mathrm{f}}$ & \multicolumn{1}{c}{$\alpha_{T}$} & $T^{-}-T^{+}$ & $q_{\mathrm{exacl}}$ & $q_{\mathrm{nos} \text { si }}$ \\
\hline 0.5 & 2.01 & 10.52 & 0.095 & 2.01 & 1.82 \\
0.6 & 1.73 & 9.95 & 0.093 & 1.73 & 1.57 \\
0.7 & 1.50 & 10.74 & 0.087 & 1.50 & 1.37 \\
0.8 & 1.31 & 13.24 & 0.077 & 1.31 & 1.20 \\
\hline
\end{tabular}

temperature gradient in the $y$-direction, while in the $x$-direction the periodic boundary conditions are used. A schematic of the computational domain is shown in Fig. 1(d). When the fluid bounds the porous medium, the solid bounding medium in Fig. $1(\mathrm{~d})$ is replaced by the fluid. Isothermal contours are shown in Fig. 3(d) for the transverse heat conduction with $k_{\mathrm{s}_{\mathrm{h}}} / k_{\mathrm{f}}=1$. For the evaluation of $k_{\mathrm{c}, 1}(y) / k_{\mathrm{r}}$, we imposed a temperature gradient in the $x$-direction similar to the one cell model used to compute the bulk effective conductivity in Section 4 . The adiabatic boundary conditions are used at the lower and upper bound-

Table 4. Effect of the particle conductivity on the slip coefficient for an in-line arrangement of circular cylinders and a fluid bounding medium $(\varepsilon=0.5)$

\begin{tabular}{rrrrr}
\hline$k_{\mathrm{s}} / k_{\mathrm{r}}$ & \multicolumn{1}{c}{$\alpha_{T}$} & $T^{-}-T^{+}$ & $q_{\mathrm{cxict}}$ & $q_{\text {nuslir }}$ \\
\hline 5 & 10.52 & 0.095 & $2.0 \mathrm{I}$ & 1.82 \\
10 & 7.69 & 0.130 & 2.42 & 2.11 \\
50 & 5.73 & 0.174 & 2.92 & 2.41 \\
\hline
\end{tabular}


aries. Isothermal contours for the longitudinal conduction are shown in Fig. 3(e) for $k_{\mathrm{s}_{\mathrm{h}}} / k_{\mathrm{r}}=1$.

5.2.I. Transterse effectice conductivity. Here we begin by reporting on the results for $k_{s_{\mathrm{n}}} / k_{\mathrm{f}}=1$. Using the volume-averaged temperature distribution and the imposed temperature gradient in the $y$-direction, $k_{\mathrm{c} \perp}(y) / k_{r}$ is cvaluated using the $y$ ' component of equalions (26) and (27), i.e.

$$
\frac{k_{\mathrm{c} \perp}\left(y^{\prime}\right)}{k_{\mathrm{r}}} \frac{\partial\langle T\rangle_{1}}{\partial \mathrm{y}^{\prime}}=\left\langle\begin{array}{c}
\left.k \frac{\partial T}{k_{\mathrm{r}}} \frac{\partial}{\partial y}\right\rangle_{\mathrm{r}} \\
\hline
\end{array}\right.
$$

For the same reasons discussed in Section 3, the volumeaveraged heat flux in the $\mathrm{l}$-direction is independent of $l$, and is given by

$$
\left\langle-\frac{k}{k_{\mathrm{r}}} \frac{\partial T}{\partial y}\right\rangle_{1}=\left\langle q_{y}\right\rangle_{\mathrm{A}_{1}}\left(y=-L_{1}\right) .
$$

Then, $k_{\mathrm{e}-1}(y) / k_{\mathrm{r}}$ is given by

$$
\frac{k_{\mathrm{a} 1}\left(y^{\prime}\right)}{k_{\mathrm{r}}}=\frac{-\left\langle q_{y}\right\rangle_{A_{1}}\left(y^{\prime}=-L_{1}\right)}{\frac{\partial\langle T\rangle_{1}}{\partial y^{\prime}}(y)} .
$$

The distribution of the effeclive conductivity com- puted using equation (49) is used in equation (1) to confirm the validity of the model given by equation (I) and to justify the use of the variable averaging volume near the interface. This is done for the same temperature boundary conditions as the pointwise solution for $\varepsilon=0.5$ and $k_{\mathrm{s}} / k_{\mathrm{f}}=50$. The agreement in heat flux between the pointwise solution and the solution to equation ( 1 ) is very good (i.e. $0.1 \%$ difference). Thus, the use of variable averaging volume in conjunction with equation (1) near the interface is justified.

The results for $k_{\mathrm{c} \perp}(\mathrm{y}) / k_{\mathrm{f}}$ for several $k_{\mathrm{v}} / k_{\mathrm{r}}$ and $\varepsilon=0.5$ are shown in Fig. 4(a). Note that $k_{\mathrm{e} \perp}(\mathrm{l}) / k_{\mathrm{r}}$ depends strongly on $k_{\mathrm{s}} / k_{\mathrm{r}}$ for $-0.5<\mathrm{y}<0$. This is due to the higher solid phase fraction (i.e. lower porosity) in the averaging volume near the interface. In Fig. 4(b) the dependence of $k_{\mathrm{e}}(y) / k_{\mathrm{r}}$ on the porosity is demonstrated. As the porosity of the porous medium increases, the solid fraction decreases in the bulk and near the interface leading to a lower local effective conductivily. The effect of porosity near the interface is also evident in the experiments of Yagi and Kunii ; however, their results are for $k_{s_{\mathrm{h}}} / k_{\mathrm{f}}>1$.

The computed local effective conductivity at the (a)

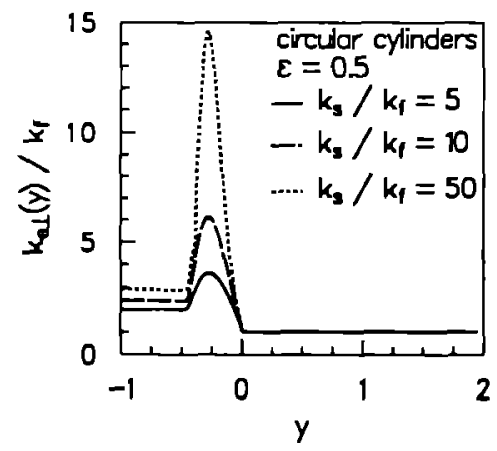

(c)

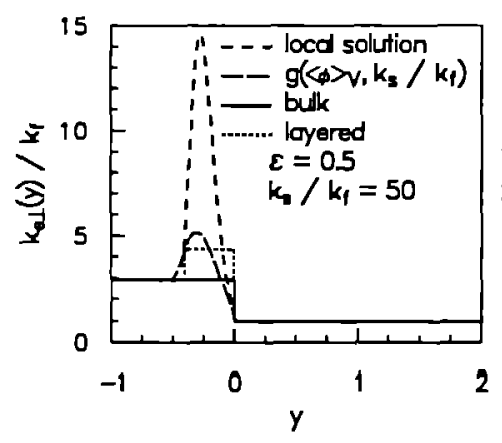

(b)

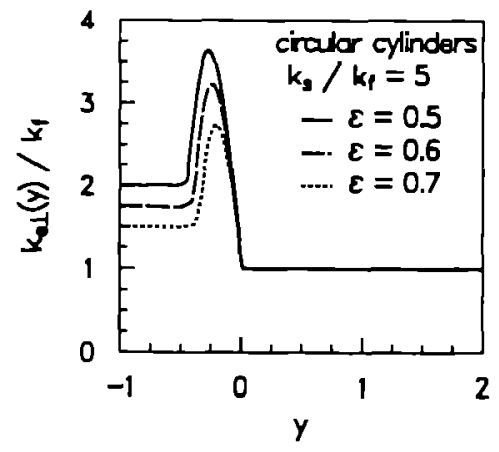

(d)

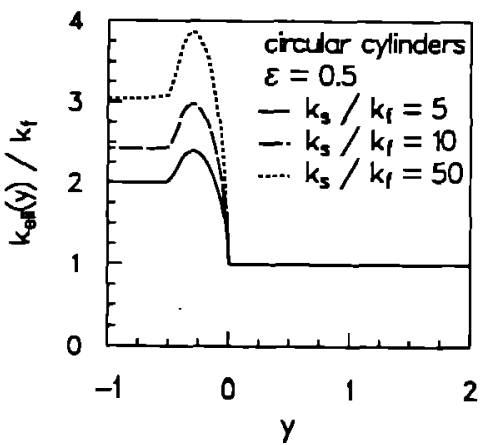

FIG. 4. (a) Distribution of the local transverse effective conductivity for an in-line arrangement of circular cylinders for different solid conductivities $\left(\varepsilon=0.5, k_{s_{\mathrm{b}}} / k_{\mathrm{f}}=1\right)$, (b) effect of the bed porosity, for an in-line arrangement of circular cylinders, on the distribution of the local transverse effective conductivity $\left(k_{\mathrm{s}} / k_{\mathrm{f}}=5, k_{\mathrm{s}} / k_{\mathrm{f}}=1\right)$, (c) distribution near the interface for the transverse effective conductivity using different models $\left(k_{\mathrm{s}} / k_{\mathrm{f}}=50, \varepsilon=0.5\right.$ ), and (d) distribution of the longitudinal local effective conductivity

for an in-line arrangement of circular cylinders, for diferent solid conductivities $\left(\varepsilon=0.5, k_{\mathrm{s}_{\mathrm{b}}} / k_{\mathrm{f}}=\mathrm{I}\right)$. 
Table 5. Effect of the bounding solid conductivity on the transverse effective conduclivity at the interface $(\varepsilon=0.5$, $k_{\mathrm{s}}\left(k_{\mathrm{f}}=5\right.$ )

\begin{tabular}{cc}
$k_{\mathrm{sh}} / k_{\mathrm{f}}$ & $k_{\mathrm{i} 1}\left(y^{\prime}=0\right) / k_{\mathrm{i}}$ \\
\hline $\mathrm{I}$ & 1.05 \\
10 & 1.80 \\
50 & 2.01 \\
$10^{4}$ & 2.09 \\
\hline
\end{tabular}

interface (i.e. $\left.k_{x \perp}(y=0) / k_{\mathrm{f}}\right)$ is presented in Table 5 for various $k_{s \mathrm{~h}} / k_{\mathrm{l}}$. This local effective conductivity is evaluated using the derivative of the volume-averaged temperature in the porous medium. The results show that as the $k_{s_{h}} / k_{\mathrm{f}}$ increases, the $k_{\mathrm{s} \perp}(y=0) / k_{\mathrm{r}}$ increases. This is due to the smaller resistance in the bounding medium which results in a larger heat flow in the particle. The conductivity of the wall determines the distribution of the heat flux over the interface and into the porous medium. This is shown schematically in Fig. l(a) where, as depicted, more heat flows through the solid portion, especially when the solid is in direcl contact with the bounding surface. These effects also become evident by examining the slope of the volumeand area-averaged temperature distributions at the interface (i.e. $y=0$ ) in Figs. 3(a) and (b). These two cases exhibit different slopes indicating a change in the local effective conductivity.

As an alternative to the direct simulation, phenomenological models can be developed for $k_{\mathrm{c} \perp}\left(y^{\prime}\right) / k_{\mathrm{r}}$. We consider three models for the variation of the transverse effective conductivity near the interface. The first is the layered model used by Ofuchi and Kunii, where the effective conductivity within the distance of a particle radius is assumed constant and different from the bulk effective conductivity. This effective conductivity is determined using the average porosity within this distance. The second model uses the local variation of the volume-averaged porosity (or solid fraction $\langle\phi\rangle,(v))$ and uses a correlation relating the local porosity to the effective conductivity, similar to the correlation given by equation (33) and by Table I, i.e.

$$
\frac{k_{\mathrm{c} \perp}\left(y^{\prime}\right)}{k_{\mathrm{f}}}=g\left[\langle\phi\rangle_{\mathrm{H}}(\mathrm{y}), \frac{k_{\mathrm{s}}}{k_{\mathrm{f}}}\right] \text {. }
$$

The third model uses the bulk effective conductivity of the packed bed. The distribution of $k_{\mathrm{c} \perp}\left(y^{\prime}\right) / k_{\mathrm{r}}$ for the three different models are shown in Fig. 4(c) for $k_{s_{\mathrm{b}}} / k_{\mathrm{i}}$. In this figure, the exact local conductivity obtained from the volume-averaged local solution is also shown and compared to the results of the three models. These models are used to solve equation (1) for the one-dimensional version of Fig. I(d) with the boundary conditions

$$
\langle T\rangle_{1} \cdot\left(y=-L_{1}\right)=0 \text { and }\langle T\rangle_{1} \cdot(y=h)=T_{h} \text {. }
$$

Equation (1) along these boundary conditions is solved numerically using the finite-difference method. This heat flux is then normalized using the temperature difference across the interfacial cell, i.e. $\left[\langle T\rangle_{1}\left(y^{\prime}=0\right)-\langle T\rangle_{1}\left(y^{\prime}=-1\right)\right]$. This problem is equivalent to the experimental procedure where two temperatures are measured and equation (1) is integrated to find the heat flux at the interface. This approach allows us to examine the ability of the different effeclive conductivity models in predicling the heat flux when two temperatures near the interface are known. The results of these different models are shown in Table 6. The heat flux for the exacl solution is obtained from the local solution using the model shown in Fig. 1(d) and then normalized using the temperature difference across the interlacial cell as mentioned above. As expected for the exacl solution the heat flux through the cell for the case of solid bounding medium $\left(k_{s_{h}} / k_{\mathrm{r}} \gg 1\right)$ is higher than the case of the fluid bounding medium $\left(k_{\mathrm{s}_{\mathrm{h}}} / k_{\mathrm{f}}=1\right)$, when the same temperature difference is used across the last cell. Table 6 shows that the layered and the volumeaveraged porosity models give the same heat flux within a negligible numerical error for both solid and fluid bounding media. This is because these models would not distinguish between solid and fluid bounding media. The results for the heat flux in Table 6 indicate that the layered model gives a better agreement than the volume-averaged porosity model when compared to the exact solution. This is because on average the transverse effective conductivity is higher for the layered model than the porosity model as shown in Fig. 4(c). For this case of a packed bed of in-line bed of cylinders it is better to use the layered model which is easier to use since a closed form solution can be found. From these results it is obvious that using the bulk effective conductivity with no slip gives the largest error in estimating the transverse heat flux at the interface.

5.2.2. Longitudinal effective conductivity. Similar to the transverse component, the volume-averaged temperature distribution and temperature gradient in the $x$-direction are used to compute the local variation of $k_{\mathrm{c} !}(y) / k_{\mathrm{r}}$ using

$$
\frac{k_{\mathrm{c} 1}(y)}{k_{\mathrm{f}}} \frac{\partial\langle T\rangle_{v}}{\partial x}=\left\langle\frac{k}{k_{\mathrm{f}}} \frac{\partial T}{\partial x}\right\rangle_{1} \text { or } \frac{k_{\mathrm{c}, 1}(y)}{k_{\mathrm{f}}}=\frac{\left\langle\frac{k}{k_{\mathrm{f}}} \frac{\partial T}{\partial x}\right\rangle_{\mathrm{l}}}{\frac{\partial\langle T\rangle_{1}}{\partial x}} .
$$

Table 6. Comparison of the interfacial heat flux resulting from different models used for transverse effective conductivity $\left(\varepsilon=0.5, k_{\mathrm{s}} / k_{\mathrm{f}}=50\right)$

\begin{tabular}{lcc}
\hline \multicolumn{1}{c}{ Model } & $\left.q\left(k_{\mathrm{s}_{\mathrm{b}}} / k_{\mathrm{r}}\right\rangle 1\right)$ & $q\left(k_{\mathrm{s}_{\mathrm{r}}} / k_{\mathrm{r}}=1\right)$ \\
\hline Exacl & 4.43 & 3.53 \\
Layered & 3.37 & 3.35 \\
$g\left(\langle\phi\rangle_{\left.r^{\prime}, k_{\mathrm{s}} / k_{\mathrm{r}}\right)}\right.$ & 3.13 & 3.11 \\
Bulk & 2.92 & 2.92 \\
\hline
\end{tabular}


The results for $k_{\mathrm{c} \mid \mathrm{l}}(y) / k_{\mathrm{r}}$ are shown in Fig. 4(d) and the same behavior is observed as that reported for $k_{\mathrm{c} \perp}(y) / k_{\Gamma}$. However, the results in Figs. 4(a) and (d) indicate that $k_{s} / k_{r}$ does not influence the local value of $k_{\mathrm{e}: \mathrm{l}}(y) / k_{\mathrm{r}}$ as much as $k_{\mathrm{c} \perp}(y) / k_{\mathrm{r}}$. These results show that there is a significant difference between the two components which show the extent of the anisotropy in $\mathbf{K}_{c} / k_{r}$ near the interface. From these results, we notice that the error in the longitudinal heat flux, introduced by assuming a constant longitudinal effective conductivity, is smaller than that for the transverse heat flux. The three models used above for the transverse case are also used here and the results for the heat flux through the interfacial cell are compared to the exact solution. The cell-averaged dimensionless heat flux through the interfacial cell is given by

$$
\bar{q}_{\mathrm{x}}=\int_{-1}^{0} \frac{k_{\mathrm{c}}(y)}{k_{\mathrm{f}}} \frac{\mathrm{d}\langle T\rangle_{1}}{\mathrm{~d} x} \mathrm{~d} y .
$$

Because the temperature is normalized using the temperature difference across one unit cell, the gradient in the $x$-direction of the volume-averaged temperature is unity. Thus, the $x$-component of the heat flux in equation (53) is equal to the integral of the local longitudinal effective conductivity. Comparison with the exact solution reveals that the bulk effective conductivity gives the most accurate prediction of the heat flux. As expected, this model underestimates the heat flux by $6 \%$ while the layered and the volumeaveraged porosity models overestimate by 8 and $15 \%$, respectively. This shows that the layered model which is the choice model for the transverse case is not satisfactory for the longitudinal case.

\section{SUMMARY}

The problem of heat conduction in porous media is treated for the bulk of a two-dimensional porous medium and also near its bounding surfaces. For the bulk effective conductivity, it is found that at low porosities (i.e. $0.4 \leqslant \varepsilon \leqslant 0.7$ ) the effects of the particle shape (square or circular cylinders) and arrangement (in-line or staggered) are significant.

For the slip boundary condition, we found that the slip coefficient $\alpha_{T}$ depends on $\varepsilon, k_{\mathrm{s}} / k_{\mathrm{r}}$, and $k_{\mathrm{s}_{\mathrm{h}}} / k_{\mathrm{r}}$. The slip in temperature is more significant when the particles are in direct contact with a solid bounding medium having a larger conductivity than the fluid.

For the no-slip boundary condition, we examined the distribution of the local effective conductivity in the longitudinal and transverse directions. We found that the local effective conductivity tensor depends strongly on the bulk and the local porosity, and on $k_{\mathrm{s}} / k_{\mathrm{r}}$ and $k_{\mathrm{s}_{\mathrm{b}}} / k_{\mathrm{r}}$. We also found that near the interface the variation of $k_{\mathrm{c} \perp} / k_{\mathrm{r}}$ is much larger than $k_{\mathrm{e} /} / k_{\mathrm{r}}$ and examined different models for these transverse and longitudinal components of the effective conductivity tensor. It is found that the layered model which uses the average porosity at the vicinity of the interface results in a better prediction of the heat flux compared to the variable $k_{\mathrm{c} \perp}(y) / k_{\mathrm{r}}$ obtained from the $k_{\mathrm{c}} / k_{\mathrm{r}}$ correlations. It is also shown that using the bulk $k_{\mathrm{c}} / k_{\mathrm{r}}$ for the transverse effective conductivity causes a significant error in the heat flux. However, for the prediction of longitudinal heat flux the constant $k_{\mathrm{c}} / k_{\mathrm{i}}$ model is the most accurate, while the layered model is the least accurate

In the near future we plan to examine the nonuniformity and anisotropy of the dispersion tensor near the interface. There, because of the nonuniformity of the flow field, the slip in the temperature is expected to be more pronounced. The effective conductivity tensor obtained here will be used in the evaluation of the dispersion tensor.

\section{REFERENCES}

I. M. Sahraoui and M. Kaviany, Slip and no-slip velocity boundary conditions at interface of porous, plain media, Int. J. Heat Mass Transfer 35, 927-943 (1992).

2. S. Yagi and D. Kunii, Studies on heat transfer near wall surface in packed beds, A.I.Ch.E. J/6, 97-104 (1960)

3. K. Ofuchi and D. Kunii, Heat trans Ter characterislics of packed beds with stagnant huids. Int. J. Heat Mass Transfer 8, 749-757 (1965).

4. A. Matsuura, Y. Hitaka, T. Akehata and T. Shirai, Apparent wall heat transfer coefficient in packed beds with downward co-current gas-liquid flow, Heat Transfer-Jap. Res. 8. 53-60 (1979).

5. G. S. Beavers and D. D. Joseph, Boundary conditions at a naturally permeable wall, $J$. Fluid Mech. 30, 197 207 (1967).

6. M. Prat, On the boundary conditions at the macroscopic level, Transp. Porous Media 4, 259-280 (1989).

7. M. Prat, Modeling of heat transfer by conduction in a transition region between a porous medium and an external fluid, Transp. Porous Media 5, 71-95 (1990).

8. G. K. Batchelor and R. W. O'Brien, Thermal or electrical conduction through a granular material. Proc. $R$. Soc. (London) A355, 313-333 (1977).

9. L. Nayak and C.-L. Tien, A statistical thermodynamic theory for coordination-number distribution and effeclive thermal conductivity of random packed beds, $I n t . J$. Heat Mass Transfer 21, 669-676 (1978).

10. M. Kaviany, Principles of Heal Transfer in Porous Media. Springer, New York (1991)

II. I. Nozad, R. G. Carbonell and S. Whitaker, Heat conduction in multi-phase systems I: theory and experiments for two-phase systems, Chem. Engng Sci. 40, 843855 (1985).

12. D. R. Shonnard and S. Whitaker, The effective thermal conductivity for a point contact porous medium: an experimental study, Int. J. Heat Mass Transfer 32, 503512 (1989).

13. A. E. Saez, J. C. Perfetti and I. Rusinek, Prediction of effective diffusivities in porous media using spatially periodic models, Transp. Porous Media 6, 143-157 (1991).

14. R. G. Carbonell and S. Whitaker, Heat and mass transfer in porous media. In Fundamentals of Transport Phenomena in Porous Media (Edited by J. Bear and M. Y. Corapcioglu), pp. 121-198. Martinus Nijhoff, Dordrecht (1984).

15. S. Whitaker, Improved constraints for the principle of local thermal equilibrium, Ind. Engng Chen. Res. 30, 983-997 (1991). 
16. S. V. Patankar, Numerical Heat Transfer and Fluid Flow, p. 45. Hemisphere, Washington, DC (1980).

17. I. Nozad, An experimental and theoretical study of heat conduction in two- and three-phase systems, Ph.D. thesis, University of California, Davis, California (1983).

18. E. F. Jaguaribe and D. E. Beasley, Modeling of the effective thermal conductivity and diffusivity of a packed bed with stagnant fluid, Int. J. Heat Mass Transfer 27, 399-407 (1984).

19. W. G. Gray, Local volume averaging of multiphase systems using a non-constant averaging volume, Int. J. Multiphase Flow 9, 755-76I (1983). 